\title{
Flight Testing a Linear Parameter Varying Control Law on a Passenger Aircraft
}

\author{
Christian Weiser*, Daniel Ossmann ${ }^{\dagger}$, Richard Kuchar ${ }^{\ddagger}$, Reiko Müller ${ }^{\S}$, Daniel MilzI , and Gertjan Looye \\ Institute of System Dynamics and Control, German Aerospace Center (DLR), 82234 Weßling, Germany
}

To improve performance and efficiency of future aircraft generations, multi-objective control design problems considering effects such as over-actuation and lowly damped flexible modes arise from new materials in the wing design. A candidate method, which delivers a solution to this problem for the whole flight envelope is linear parameter varying (LPV) control synthesis. It already incorporates the controller scheduling in the synthesis process, guaranteeing stability and robustness over the entire scheduling envelope, and enables intuitive multi-objective, multiple-input multiple-output (MIMO) controller designs. To prove the concept of LPV controllers in a realistic environment, the flight test campaign results of LPV inner loop control laws on a Cessna Citation II (550) aircraft are presented in this paper. The implemented inner loop controllers are inspired by classical flight controllers used on state-of-the-art fly-by-wire airliners. The longitudinal motion is augmented with load-factor command and the lateral motion controller, which is inherently of MIMO type, features a roll rate command with attitude hold behavior. The control laws are validated in flight by the pilot with respect to functionality, flying and handling qualities. Furthermore, auto generated input signals are used to excite the aircraft without pilot in the loop to allow for a comparability with simulation results.

\section{Introduction}

The design of flight control laws for contemporary transport aircraft is still mostly relying on classical control methods, such as linear proportional-integral-derivative (PID) controllers. These are, in a second design step, scheduled to provide a control system for the full flight envelope. One advanced control design technique which showed already promising results in flight is linear parameter varying (LPV) control [1, 2]. In addition to the capability to include the controller scheduling in the design [3], the technique can provide good robustness against uncertainties due to its closed loop shaping design approach. In this sense, the LPV design can be seen as an extension of the $\mathrm{H}_{\infty}$ design method, which has been previously tested by the authors in a flight test campaign on an unmanned aerial vehicle [4]. For the validation of the LPV controller in this work, a CS 25 certified Cessna Citation II is chosen as controller testbed. On this aircraft, extensive experience of testing various fly-by-wire (FBW) control laws has been gained within previous test campaigns for (incremental) non-linear dynamic inversion ((I)NDI) control and incremental backstepping (IBS) based control [5-7]. Thus, the aircraft has shown to be a very capable testbed for the validation of new control algorithms for transport aircraft.

The contribution of this paper is the development and in flight validation of an LPV controller on a passenger aircraft, i.e. the Cessna Citation II. Therefore, LPV controller synthesis is employed to derive robust and scheduled control laws for both longitudinal and lateral augmentation of the test aircraft. The controllers are then assessed in simulation and validated during flight test by the pilot. Performance design objectives are derived from classical handling quality demands for augmented flight. Besides good robustness measures, validated a-posteriori by stability margins, these performance measures are the main design goals.

Within this work, different control strategies, i.e. different commanded variables by the pilot, are selected, designed and tested on the Cessna Citation II (550) aircraft. This allows to compare different control strategies commonly used on commercial airliners. Following the design stage, these controllers have been verified on DLR's 6 degree of freedom (DOF) Robotic Motion Simulator (RMS) facility as presented in [8] as preparation to the on-board flight-testing.

\footnotetext{
*Research Assistant: Christian.Weiser@dlr.de

${ }^{\dagger}$ Professor at Munich University of Applied Sciences, former DLR, AIAA Senior Member: Daniel.Ossmann@hm.edu

†esearch Assistant: Richard.Kuchar@dlr.de

§ Research Assistant: Reiko.Mueller@dlr.de

IITrainee: Daniel.Milz@dlr.de

" Department Head: Gertjan.Looye@dlr.de
} 
In this paper we first summarize the design process and present the actual flight test results. Therefore, an overview of the applied LPV controller synthesis process is given in Sect.II For the design of the control laws, an LPV representation of the non-linear aircraft model of the Citation II is derived within Sect. III] Based on this gridded model, an LPV controller is synthesized. More details of the control design results are provided in [8]. Sect. IV]describes first the hardand software setup of the research aircraft, flight test preparation and analysis of flight test data.

\section{Linear Parameter Varying Control Design}

In this section, the LPV approach used for control design is described. $\mathrm{H}_{\infty}$ control [9] is a well known method which allows to shape the closed loop frequency response. Hence, adequate shaping weights have to be selected, which are derived based on handling and flying quality requirements [9]. Extending from this, LPV control synthesis permits to use a similar scheme for LPV models [10,11]. As advantage, the resulting controller is directly scheduled with the defined varying parameter. This guarantees stability and performance for all trajectories in $\varrho$ within the defined rate of change. Firstly, LPV models are introduced where all four state space matrices are dependent on a scheduling parameter $\varrho$, which can vary over time:

$$
G_{\varrho}:\left\{\begin{array}{l}
\dot{x}(t)=A(\varrho(t)) x(t)+B(\varrho(t)) u(t) \\
y(t)=C(\varrho(t)) x(t)+D(\varrho(t)) u(t) .
\end{array}\right.
$$

The state space matrices $A \in \mathbb{R}^{n_{x} \times n_{x}}, B \in \mathbb{R}^{n_{x} \times n_{u}}, C \in \mathbb{R}^{n_{y} \times n_{x}}, D \in \mathbb{R}^{n_{y} \times n_{u}}$ are dependent on the vector $\varrho(t), x \in \mathbb{R}^{n_{x}}$ is the $n_{x}$-dimensional state vector, $u \in \mathbb{R}^{n_{u}}$ is the $n_{u}$-dimensional input vector, and $y \in \mathbb{R}^{n_{y}}$ is the $n_{y}$-dimensional output vector. The LPV system $G_{\varrho}$ in Eq. (1) is augmented with performance weights, similar to those of $H_{\infty}$ control design approaches [9]. For the resulting augmented model $\tilde{G}_{\varrho}$, the induced $L_{2}$ norm from the disturbance inputs $d \in \mathbb{R}^{n_{d}}$ to its performance output $z \in \mathbb{R}^{n_{z}}$ over all allowed trajectories in a set $P$ is defined as

$$
\left\|\tilde{G}_{\varrho}\right\|_{\infty}=\sup _{d \in L_{2} \backslash 0, \varrho \in P} \frac{\|z\|_{2}}{\|d\|_{2}} .
$$

The induced $L_{2}$ norm measures the maximum gain of $\tilde{G}_{\varrho}$, which is equivalent to the largest gain on $L_{2}$ input signals over all frequencies and input-output directions. The LPV flight control law design herein uses the developed closed loop weighting interconnection structure illustrated in Fig. 1. With this structure the augmented plant from disturbance inputs $d=\left[\begin{array}{ll}r & d_{i} d_{o}\end{array}\right]^{T}$ to performance outputs $y$ and $z=\left[z_{e} z_{u}\right]^{T}$ is derived and used to shape the closed loop response. In the generalized plant of Fig. 1 the disturbance inputs are the reference signal $r \in \mathbb{R}^{n_{r}}$, the input disturbance $d_{i} \in \mathbb{R}^{n_{d_{i}}}$, and the output disturbance $d_{o} \in \mathbb{R}^{n_{d_{o}}}$ and thus $n_{d}=n_{r}+n_{d_{o}}+n_{d_{i}}$. Outputs are the weighted tracking error $z_{e} \in \mathbb{R}^{z_{e}}$ with $z_{e}=W_{e} e=W_{e}\left(r\left[\begin{array}{ll}W_{h} & \underline{0}\end{array}\right]-y\right)$ and the weighted control effort $z_{u} \in \mathbb{R}^{z_{u}}$ with $z_{u}=W_{u} u$. Note that $W_{h}$ often has a lower dimension as the measurement vector $y$, as only for a subset of the measurement vector reference signals are provided. To overcome the issues regarding signal dimensions in the block diagram a $n_{y} \times n_{r}$ dimensional zero matrix $\underline{0}$ is introduced as depicted in Fig. 1] The resulting generic structure serves as controller design for both the longitudinal and lateral axis. The final input-output map for the control design is given by

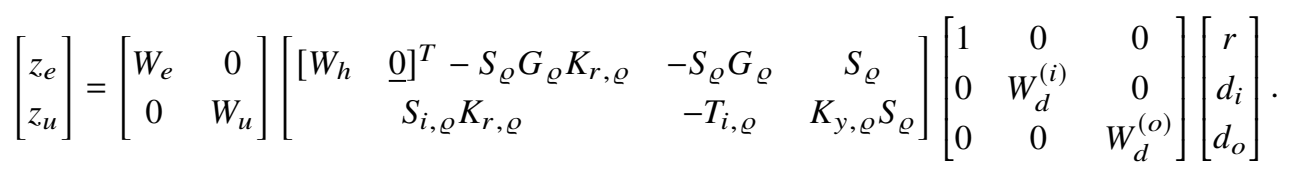

In Eq. (3) $K_{y, \varrho}$ is the feedback part and $K_{r, \varrho}$ the feed-forward part of the controller, $G_{\varrho}$ is the transfer matrix from command inputs to plant outputs. Further, $S_{\varrho}=\left(I+G_{\varrho} K_{y, \varrho}\right)^{-1}$ is the sensitivity function, and $S_{i, \varrho}=\left(I+K_{y, \varrho} G_{\varrho}\right)^{-1}$ and $T_{i, \varrho}=K_{y, \varrho} G_{\varrho}\left(I+K_{\varrho} G_{\varrho}\right)^{-1}$ are the input sensitivity and input complementary sensitivity function, respectively $T_{r, \varrho}=K_{r, \varrho} G_{\varrho} S_{\varrho}$ defines the complimentary sensitivity on reference signal. $W_{e}(s)$ and $W_{u}(s)$ are the diagonal output weighting matrices, and $W_{d_{i}}$ and $W_{d_{o}}$ are the diagonal input weighting matrices to be chosen in the design process. Note that the aircraft model $G_{\varrho}$ is scaled as proposed in [9] by input-output scaling. As indicated in Fig. 11 $W_{e}(s)$, $W_{h}(s)$ and $W_{u}(s)$ are selected as dynamic filters, while the input weightings $W_{d}^{(o)}$ and $W_{d}^{(i)}$ are constant matrices. All weightings are square with entries on the diagonal axis only. $W_{h}(s)$ denotes a handling quality filter in the form Eq. (4) and allows setting a design response in terms frequency $\omega_{h}$ and damping $\zeta_{h}$.

$$
W_{h}=\frac{\omega_{h}^{2}}{s^{2}+\omega_{h} \zeta_{h} s+\omega_{h}^{2}} .
$$




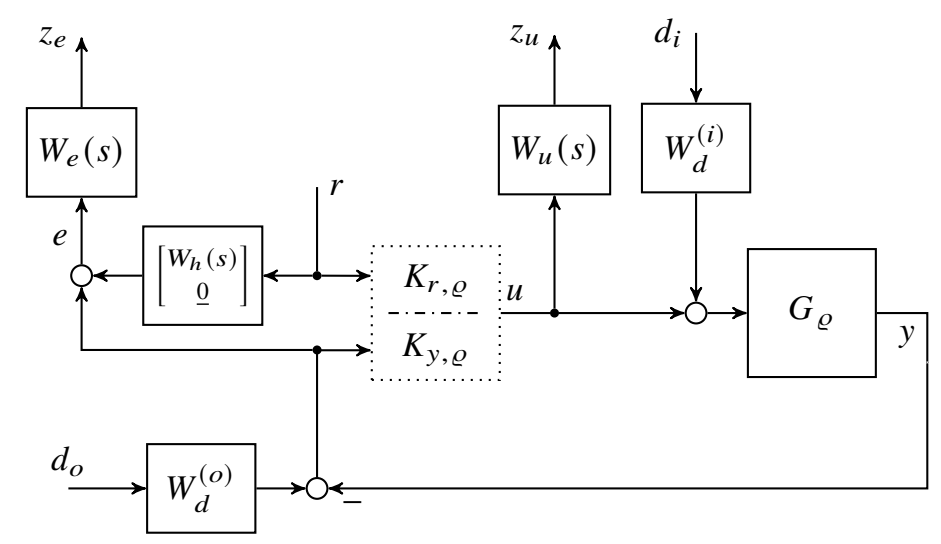

Fig. 1 Generic weighting structure used for the longitudinal and lateral controller design

The entries for the shaping filter $W_{u}$ are selected as first-order transfer functions with unit gain up to the available bandwidths $\omega_{a, i}$ for $i=1, \ldots, n$ of each control input and approximative differentiating behavior beyond that frequency. In $W_{e}(s)$ only the first element is dynamic in order to weight the tracking behavior, while all other channels are set to constant values to weight the outputs. Since integral behavior is demanded in the tracking channel, the choice for the first entry of $W_{e}$ is a first-order transfer function with approximative integral behavior up to the desired bandwidth $\omega_{b}$, which reduces the sensitivity up to this selected bandwidth. The weighting filters are selected so that the general design goals for pitch and roll control, which are taken from the level 1 flying qualities specifications will be met [12]. The demands for the flying qualities are defined in frequency domain for both longitudinal and lateral motion. An an example, the control anticipation parameter (CAP) is selected in order to design the weighting filters of the pitch controller, allowing to set a desired short period (SP) frequency. A more detailed description can be found in Sect. III.B Further, the controllers shall meet common requirements for gain and phase margins ( $6 \mathrm{~dB}, 45 \mathrm{deg})$.

The resulting parameter dependent controller

$$
K_{\varrho}=\left[\begin{array}{c|c}
A_{K}(\varrho, \varrho) & B_{K}(\varrho) \\
\hline B_{K}(\varrho) & D_{K}(\varrho)
\end{array}\right]
$$

is synthesized solving a convex optimization problem (see [13]) and depends on the scheduling vector $\varrho$ and its derivative $\dot{\varrho}$. The obtained controller minimizes the upper bound on the induced $L_{2}$-norm of Eq. 22, which is the interconnection shown in Fig. 1. For the dependence of the controller parameters on the scheduling vector a base function needs to be manually input to the design algorithm. It is meaningful to select simple base functions, as the number of their unknowns correlates to the number of unknowns in the corresponding linear matrix inequalities (LMIs) that needs to be solved during the design process.

More illustrative design examples for LPV controllers can be found in [13, 14]. The available MATLAB toolbox LPVTools [15] allows to numerically solve the LPV control design problem. 


\section{Control Law Designs}

The design process of the developed and tested LPV control laws is presented in detail in [8]. This section is meant to shortly summarize the aircraft dynamics, the control design requirements and the derived controller for the longitudinal and lateral motion.

\section{A. Aircraft Model}

The simulation model used for control design was developed based on the Delft University Aircraft Simulation Model and Analysis Tool (DASMAT) [16] and allows simulation and control design within the MATLAB / Simulink environment. The used DASMAT model was originally generated for a Cessna Model 500 Citation; nonetheless the modified simulation model shows good compliance with the Citation II [17]. It comprises of the standard 6 DoF non-linear equations and aerodynamic lookup table data based on [16]. The actuators were modeled as second order transfer functions according to [18]. The sensors are modeled as a combination of time delay, bias and noise. The basic flight control system is part of the model and features a servo controller for setting the actuator positions commanded by the flight control laws.

In order to obtain an LPV model of the Citation II aircraft, the non-linear model is trimmed and linearized over a grid of operating points, which leads to a model as defined in Eq. (1). Dynamic pressure

$$
\bar{q}=\frac{\rho}{2} V^{2}
$$

represents the scheduling variable, with $\rho$ being air density and $V$ true airspeed. This corresponds to a mapping of calibrated airspeed, which has the largest impact on the change of aircraft dynamics. For longitudinal and lateral dynamics, the LPV model of the full plant is fractioned into two models as for controller design it is appropriate to consider the two axes as decoupled [19].

The scheduling parameter dynamic pressure $\bar{q}$ can vary in the range of $\bar{q} \in[2250,6500] \mathrm{Pa}$, which corresponds to a range of calibrated airspeeds from $85 \mathrm{~m} \mathrm{~s}^{-1}$ to $120 \mathrm{~m} \mathrm{~s}^{-1}$. The parameter rate is bounded to be within $\pm 500 \mathrm{~Pa} \mathrm{~s}^{-1}$. This translates e.g. to a rate of change in airspeed of approximately $7 \mathrm{~m} \mathrm{~s}^{-2}$ when at FL 450 with initial airspeed of $90 \mathrm{~m} \mathrm{~s}^{-1}$. For the controller development, an equally spaced parameter grid with 18 points was selected and verified against a denser grid of more than 50 points. Fig. 2 depicts the poles of the open loop plant $4 \times 4$ linear time invariant (LTI) models of the aircraft at four equidistantly chosen grid points, namely dynamic pressure values of 2250,3500, 5000 and $6500 \mathrm{~Pa}$. The conjugate complex poles of the SP mode can be read with frequencies ranging from 2 to $3 \mathrm{rad} \mathrm{s}^{-1}$ and a damping ratio of approximately 0.45 . The dutch roll (DR) motion is located at a similar frequency range, but with a damping ratio of slightly less than 0.2 , this mode is only poorly damped. The roll time constant $t_{r}$ changes over a range from $0.33 \mathrm{~s}$ at highest dynamic pressure to $0.5 \mathrm{~s}$ at the lowest dynamic pressure value. The phugoid and spiral poles have large time constants and are located near the origin as seen in Fig.2.

\section{B. Longitudinal Control Laws}

For the longitudinal control augmentation load factor command $n_{z, \mathrm{cmd}}$ is selected as tracking reference for control design. The designed controller commands the maneuver load factor of the aircraft set by the pilot's pitch control input. As the $n_{z, b}$ sensor measurement includes gravity, the feedback needs to be corrected by the aircraft's pitch and roll attitudes $(\Theta, \Phi)$ :

$$
n_{z, \operatorname{man}}=n_{z, \mathrm{cg}} \frac{\cos (\Theta)}{\cos (\Phi)}
$$

This corrected value $n_{z \text {,man }}$ results in a value of $1 \mathrm{~g}$, independent of the aircraft's attitude and flight path. This design approach has the advantage that a zero command input results in attitude hold behavior which is convenient for augmented flight. Therefore, neutral stick input is related to a $1 \mathrm{~g}$ flight with flight path angle hold. As the test aircraft is not equipped with an auto-throttle system, manual adjustment of the throttle is mandatory to keep the airspeed and dynamic pressure inside the test envelope bounds. In order to obtain a stable and controllable plant, control design only considers elevator input together with the SP model, with angle of attack and pitch rate as states and load factor $n_{z}$ as additional plant output. Due to the missing auto-throttle, the $4^{\text {th }}$ order longitudinal model is uncontrollable which is why the phugoid poles are not considered for controller synthesis, and instead the $2^{\text {nd }}$ order SP model is being used. When integrating the controller into the full model, the result will be a phugoid motion with two real poles of which one may be in the unstable region, but with a time constant larger than 30 seconds. This leads to the requirement of manual 


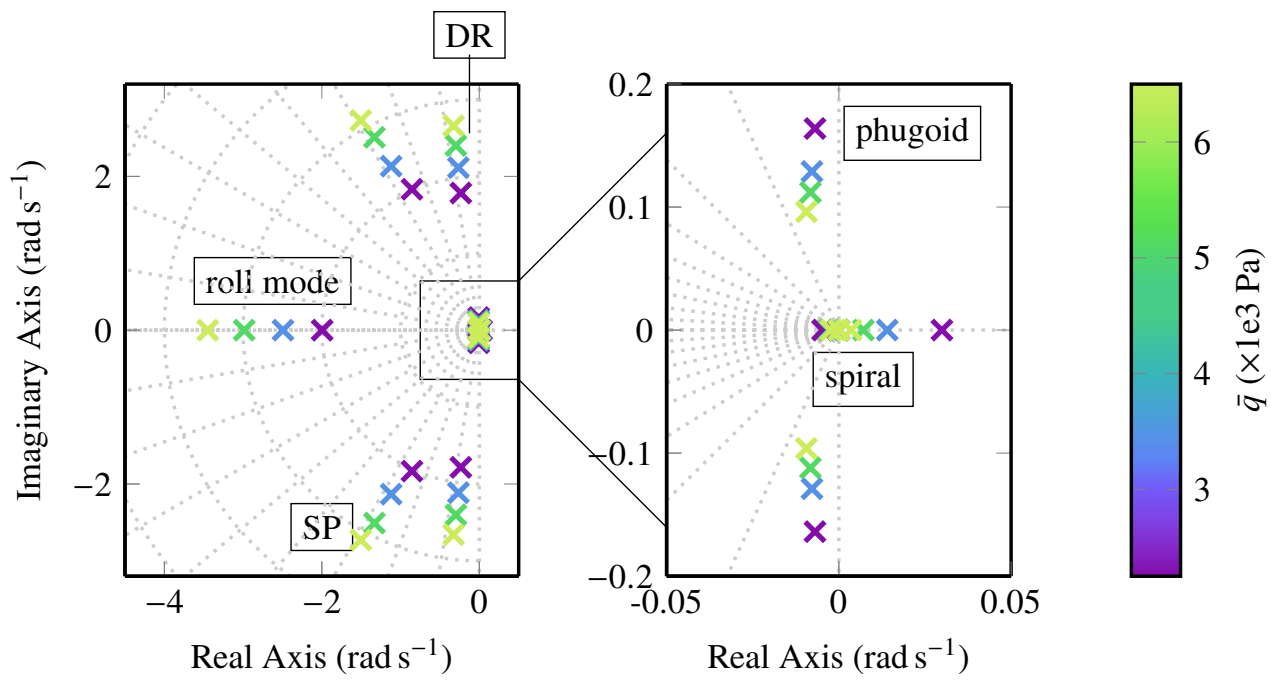

Fig. 2 Pole map of the open loop LTI models at 4 different values of $\bar{q}$, showing the $4^{\text {th }}$ order longitudinal model together with the $4^{\text {th }}$ order lateral model.

adjustment of thrust settings accordingly to avoid stall or overspeed regions of the envelope.

The limits of the controlled output are $\pm 0.3 \mathrm{~g}$ for the commanded load factor. The design process for the maneuver load factor requires $r=n_{z \text {,man }}$, as the tracking reference variable. The measured outputs are the load factor in body z-axis and the pitch rate of the aircraft. Design goals for the longitudinal motion are defined in [12] via the control anticipation parameter, as well as a minimum SP damping of $\zeta=0.3$ and an optimal SP damping value of $\zeta=0.7$.

The CAP describes the relation of the immediate pitch acceleration to a control input and the 'stationary' load factor which will be the result of this maneuver. The CAP is defined as

$$
\mathrm{CAP}=\frac{\omega_{S P}^{2}}{n_{z, \alpha}},
$$

where $\omega_{S P}$ is the SP frequency and $n_{z, \alpha}$ the (stationary) load factor resulting from a change in angle of attack. The value of $n_{z, \alpha}$ is characteristic for each aircraft, thus only $\omega_{S P}$ can be influenced. The CAP has a goal value of one, which means that the pilot is able to determine the amount of stick input needed for a certain load factor easily from the pitch acceleration $\dot{q}_{0}$ which is a direct result of an elevator deflection $\eta$, whereas the load factor $n_{z}$ takes time to build up as it has two integral stages in between:

$$
\eta \rightarrow \dot{q} \stackrel{\int}{\rightarrow} q \rightarrow \dot{\alpha} \stackrel{\int}{\rightarrow} \alpha \rightarrow n_{z},
$$

where $\eta$ is elevator deflection, $q$ pitch rate and $\alpha$ angle of attack. In case of a CAP smaller than one, the pitch acceleration after a control input is low and the pilot will have the tendency of underestimating the stationary load factor, thus giving higher pitch input as needed and overshooting the desired load factor. The handling quality level 1 boundaries and results of the chosen controller design for load factor control can be seen in Fig. 3 The resulting CAP is in the level 1 region close to the optimal value for the whole design envelope which was in this case achieved with a constant handling quality filter using a frequency of $1.75 \mathrm{rad} \mathrm{s}^{-1}$ and a damping ratio of 0.8 . The load factor longitudinal controller features 12 states and shows integrating behavior up to a frequency of $2 \mathrm{rad} \mathrm{s}^{-1}$.

The time domain results of the linear $n_{z}$ command design exhibit rise times smaller than $4 \mathrm{~s}$ for low and smaller than $2 \mathrm{~s}$ for high dynamic pressures. In terms of robustness, the MIMO disk margin has a minimum at the upper end of the parameter envelope with values of $6.4 \mathrm{~dB}$ and $41 \mathrm{deg}$ respectively, which can be considered as sufficient. The margins listed for the load factor control in Table 1 are a comparison of standard gain/phase/delay margins of loop cuts at sensor / actuator signal(s) with more significant disk margins and robustness margins used in [20]. The disk margin is computed according to [21] and values for the phase of the disk margin vs. the scheduling parameter of the controller are depicted in Fig. 3. The drop of the margins for the high dynamic pressure region calls for a careful flight testing at 


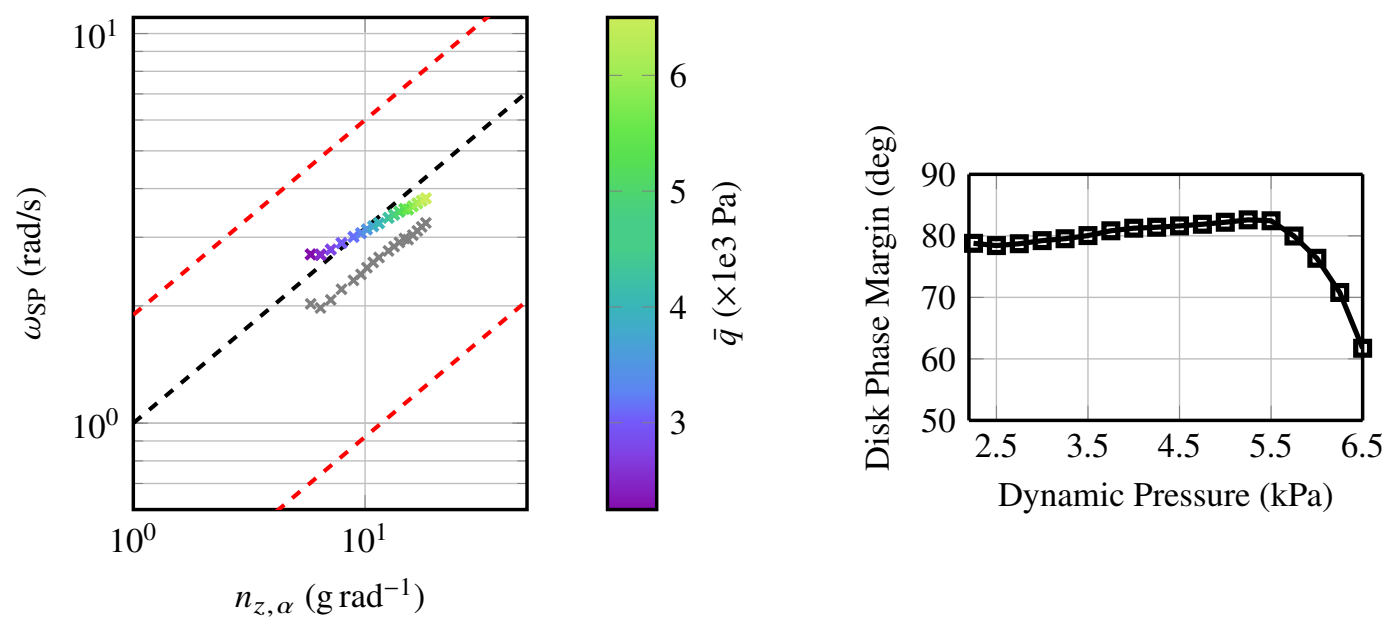

Fig. 3 Left hand side: CAP for the $n_{z}$ control law throughout the flight envelope of the closed loop compared to the open loop $($ ). Additionally, the ideal CAP value of $1(--)$ and level 1 flying qualities region boundaries [-- according to [12] are depicted. Right hand side: disk margin (phase) of the load factor controller w.r.t. scheduling parameter (dynamic pressure).

Table 1 Minimum margins of the load factor controller.

\begin{tabular}{llll}
\hline Margin Type & $\begin{array}{l}\text { Frequency } \\
(\mathrm{rad} / \mathrm{s})\end{array}$ & Value & \\
\hline Gain Margin & 247 & $13 \mathrm{~dB}$ & \\
Phase Margin & 0.68 & $83 \mathrm{deg}$ & \\
Delay Margin & 1.0 & $1.44 \mathrm{~s}$ & \\
Disk Margin & 117 & $11.8 \mathrm{~dB}$ & eq. 62 deg Phase Margin \\
MMIO $^{1}$ & 0.81 & $6.4 \mathrm{~dB}$ & eq. 41 deg Phase Margin \\
\hline
\end{tabular}

${ }^{1}$ Multi-Input-Multi-Output Margin

the end of the envelope. As the maneuverability of the experimental flight control system (FCS) is limited by relatively moderate power of the pitch servo, most maneuvers are flown in a low dynamic pressure region.

When looking at frequencies, it can be noted that the frequency of the minimum disk margins in Fig. 3 is located several magnitudes above the system bandwidth. This makes it uncritical even in the case of exceeding the flight envelope.

\section{Lateral Control Laws}

For the lateral inner loop, the roll rate $p$ and angle of sideslip $\beta$ are selected as control variables. In the chosen setup, the experimental sidestick on the right hand pilot's seat allows to command a roll rate up to a bank angle of $27 \mathrm{deg}$, where constant stick input is needed for higher bank angles (bank angle control). The maximum bank angle of 35 deg shall never be exceeded and will return to $27 \mathrm{deg}$ upon stick release. The aircraft is not equipped with a yaw input column on the experimental FCS, thus the yaw axis is assessed via differential thrust settings.

For the inner loop control laws designed in this section, flying quality specifications for lateral axis [12] request a maximum time of $2 \mathrm{~s}$ for a $30 \mathrm{deg}$ bank angle change. Thus, a roll rate limit of $p_{\max }=15 \mathrm{deg} \mathrm{s}^{-1}$ is introduced to fulfill this requirement. The roll time constant $t_{r}$ shall be less than one second, which allows a quick response to pilot's sidestick input. For the DR mode, specifications request a minimum damping of 0.08 and a minimum frequency of $0.4 \mathrm{rad} \mathrm{s}^{-1}$ as well as the product of damping and frequency to be at least $0.15 \mathrm{rad} \mathrm{s}^{-1}$.

The handling quality filter for roll rate is chosen as a second order filter in the form of Eq. 4 with a frequency of $\omega_{S P}=5 \mathrm{rad} \mathrm{s}^{-1}$ and a damping of $\zeta=0.9$, to provide tracking of the commanded roll rate. The same form of filter, with a lower corner frequency, is chosen for the angle of sideslip channel. The performance weight on the tracking 
Table 2 Minimum margins of the lateral control loop.

\begin{tabular}{|c|c|c|c|}
\hline Margin Type & $\begin{array}{l}\text { Frequency } \\
(\mathrm{rad} / \mathrm{s})\end{array}$ & Value & \\
\hline gain margin $\left(\mathrm{I}^{1}\right)$ & 1.38 & $11.5 \mathrm{~dB}$ & \\
\hline phase margin (I) & 0.45 & $73.8 \mathrm{deg}$ & \\
\hline gain margin $\left(\mathrm{O}^{2}\right)$ & 1.72 & $10.8 \mathrm{~dB}$ & \\
\hline phase margin $(\mathrm{O})$ & 0.47 & $70.2 \mathrm{deg}$ & \\
\hline delay margin (I/O) & 1.72 & $0.54 \mathrm{~s}$ & \\
\hline disk margin (I) & 1.21 & $11.3 \mathrm{~dB}$ & $\begin{array}{l}\text { (equals } 59.5 \text { deg Phase Mar- } \\
\text { gin) }\end{array}$ \\
\hline disk margin $(\mathrm{O})$ & 1.31 & $10.0 \mathrm{~dB}$ & $\begin{array}{l}\text { (equals } 54.8 \text { deg Phase Mar- } \\
\text { gin) }\end{array}$ \\
\hline $\mathrm{MMIO}^{3}$ & 1.35 & $4.2 \mathrm{~dB}$ & $\begin{array}{l}\text { (equals } 26.8 \text { deg Phase Mar- } \\
\text { gin) }\end{array}$ \\
\hline
\end{tabular}

error for both channels is chosen as a transfer function with integrating behavior up to a frequency of $12 \mathrm{rad} / \mathrm{s}$, which is sufficiently lower than the actuator bandwidth of $30 \mathrm{rad} / \mathrm{s}$. The actuator weights are selected to have differentiating behavior above the actuator bandwidth to suppress control action above the actuator bandwidth. All other weights are set to constant. For both reference variables, nearly parameter independent behavior has been achieved which results in similar aircraft responses in roll axis over the whole envelope. Roll rate is tracked up to a frequency of 8 rad s$^{-1}$ and angle of sideslip up to $1 \mathrm{rad} \mathrm{s}^{-1}$, above this frequency tracking accuracy diminishes due to higher prioritized yaw damping. The lateral controller has a number of 20 internal states.
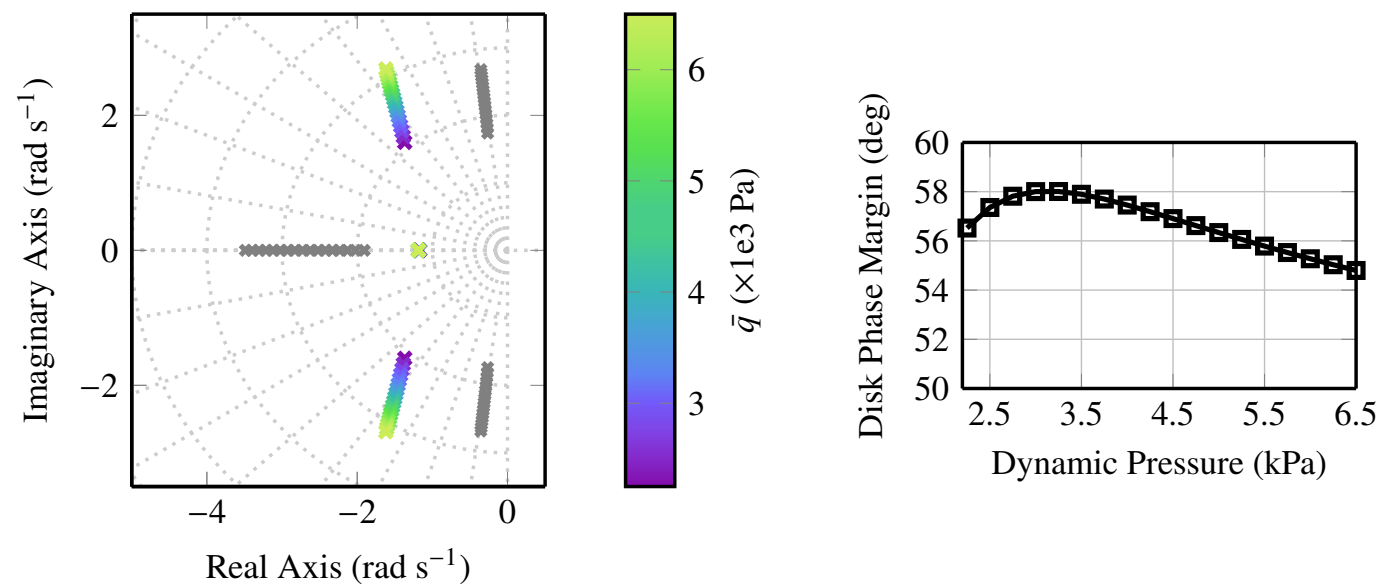

Fig. 4 Left hand side: poles of lateral open loop and closed loop $x$. DR shows increased damping ratio for the closed loop and roll time constant is clipped to a constant value for the whole parameter envelope in closed loop. Right hand side: minimum disk margin (phase) of the lateral control loop w.r.t. scheduling parameter.

The left hand part of Fig. 4 shows the closed loop poles of the controlled LPV system versus the poles of the uncontrolled LPV plant. According to the previously explained tracking behavior, it can be clearly seen that a unique roll time constant for the full envelope is generated. This ensures similar times for the build-up of roll rate independent of the dynamic pressure, thus the pilot can expect similar behavior of the aircraft's roll axis without considering the actual airspeed and altitude. Moreover, an increased damping of the DR motion is achieved when comparing the closed loop with open loop poles.

On the right hand side plot of Fig. 4, the phase of the minimal disk margin of the lateral controller is depicted. Table 
2 shows a comparison of classical gain / phase margins and the robustness margin computations introduced in Sect. III.B. where sufficient robustness with a minimum of $4.2 \mathrm{~dB}$ against simultaneous variations in all channels is ensured.

\section{Flight Test}

This section is meant to give a brief overview about the Cessna Citation research aircraft used for flight testing as well as an introduction to the software architecture used for the flight tests. Additionally, the selected maneuvers and the motivation for their selection are discussed followed by analysis of flight test data.

\section{A. Test aircraft and control system}

The Cessna Citation PH-LAB (see Fig. 5), jointly operated by TU Delft and the Dutch Aerospace Center (NLR) serves as a multi-functional research platform. The aircraft is certified according to CS25 specifications for large airplanes and equipped with a conventional, fully reversible flight control system providing a fix-geared link between the pilot's controls and the control surfaces of the aircraft. Additionally, an autopilot which has authority over the primary flight controls (elevator, aileron, rudder) is available. The test aircraft is equipped with an experimental FBW system [22], which uses the autopilot servos as control actuators. This setup has been thoroughly tested and as well certified under CS 25 [23]. In addition, a flight test instrumentation system [24] including further sensors (angle of attack, sideslip etc.) is available for data acquisition and logging. The hardware setup is described in more detail in Refs [5, 22, 24].

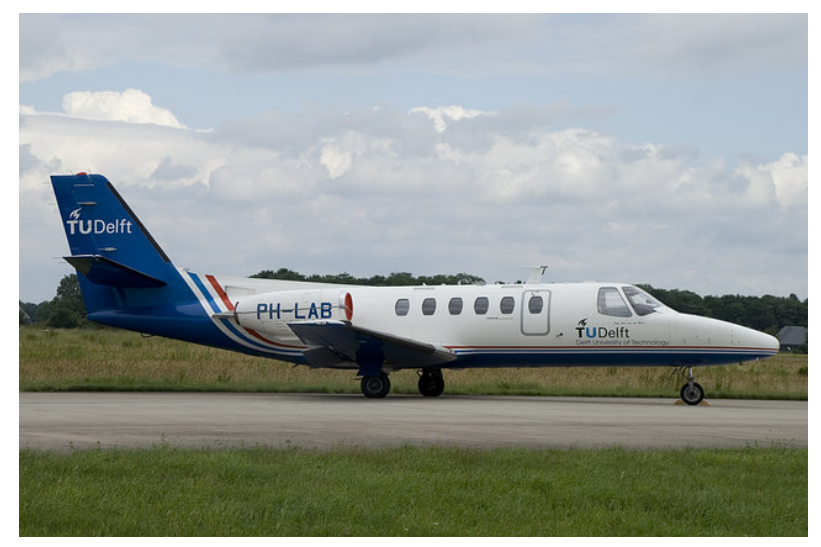

Fig. 5 Cessna Model 550 Citation II Research Aircraft PH-LAB [25].

A linear rate command attitude hold (RCAH) outer loop is added to the lateral LPV inner loop control system. As shown in Fig. 6, the command module, which contains the described RCAH outer loop, translates the stick signal which is given either by the pilot or the flight test engineer to the reference signals needed for the LPV inner loop. The described control system setup has been tested in previous experiments on the Citation aircraft [5-7] and is consequently reused in the shown flight tests. The attitude hold option can be deactivated within the command module. As a result, it is possible to activate either the RCAH mode, which resembles an Airbus-like system behavior, or rate-command-only mode which corresponds to a control philosophy in which the pilot needs to correct changes in attitude due to external disturbances. The output signals of the LPV control feed the FBW servo control system [18], which uses position feedback from the control surfaces to set the demanded deflections.

\section{B. Test Cases and Test Execution}

The flight test scenarios are described in Table 3 The test campaign comprised two test flights which were carried out in calm environmental conditions (no turbulence, constant wind of low magnitude) within a military testing airspace over the southern Netherlands (see Fig. 7). Both flights followed a similar scheme which involved after successful activation of the controller followed by careful maneuvers by the experimental pilot, slowly expanding to the full range of the maximum control authority including sweeps through the full range of the reference signal.

In order to evaluate the tracking behavior and stability (rise time, settling time) the pilot was asked to command step inputs with small amplitudes followed by the same maneuver being repeated with automated input signals. Since 


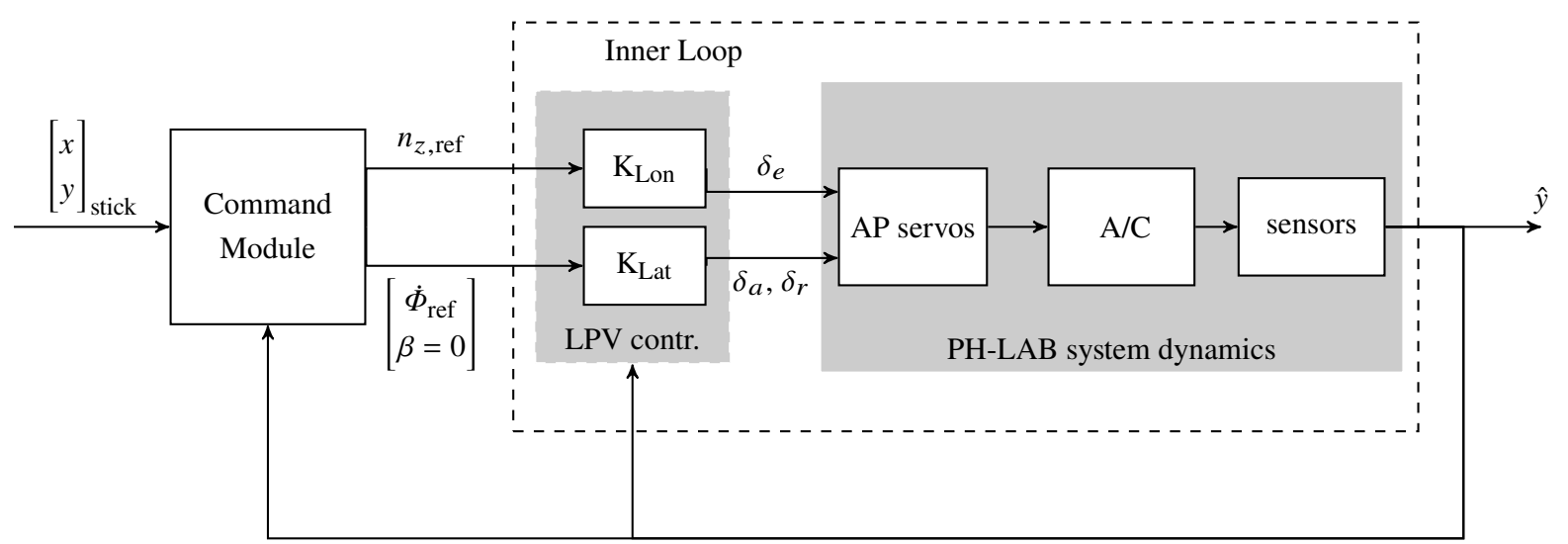

Fig. 6 Control system architecture.

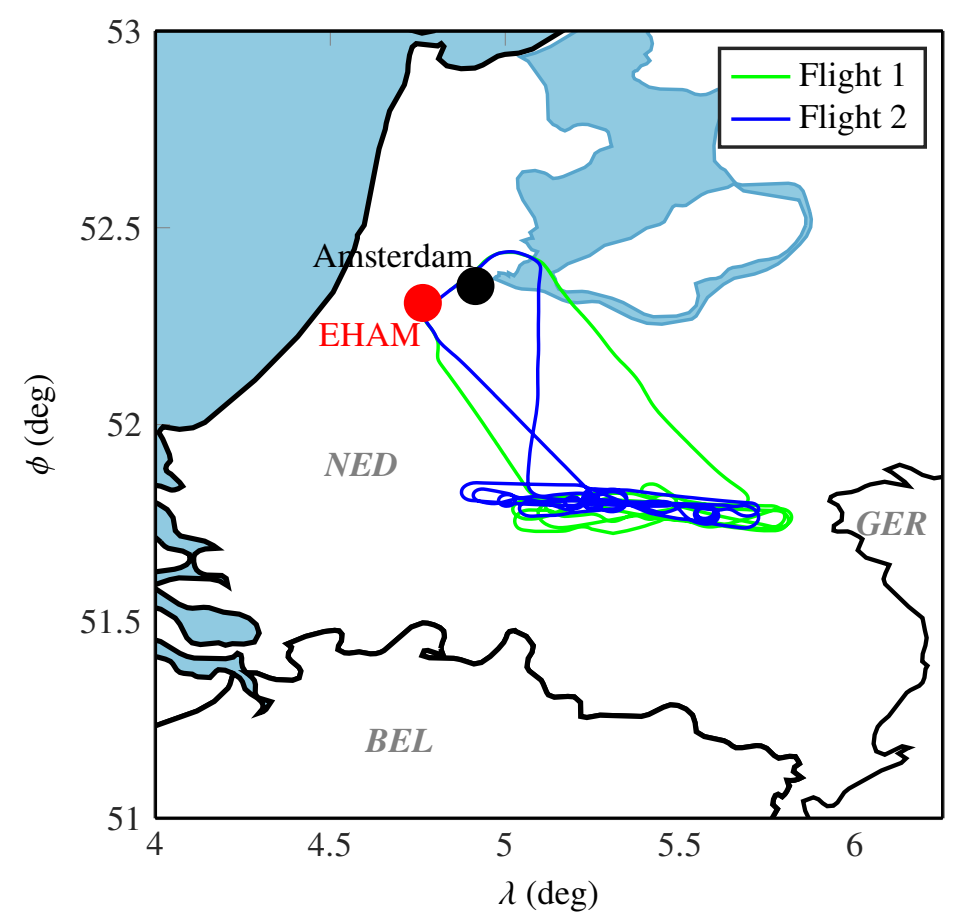

Fig. 7 Flight Trajectories. 
Table 3 Overview of flight test maneuvers

\begin{tabular}{|c|c|c|}
\hline Maneuver & Synopsis & Objective \\
\hline Steady flight & $\begin{array}{l}\text { No control inputs, controller maintains system } \\
\text { state at time of activation }\end{array}$ & $\begin{array}{l}\text { Validate controller stability, check for } \\
\text { transient free activation }\end{array}$ \\
\hline Pilot inputs & $\begin{array}{l}\text { Pilot commands roll or pitch, extending from } \\
\text { small inputs to full control authority }\end{array}$ & $\begin{array}{l}\text { Validate system controllability and track- } \\
\text { ing performance }\end{array}$ \\
\hline Attitude control & $\begin{array}{l}\text { Pilot commands roll angles beyond } 27 \mathrm{deg} \text { of } \\
\text { roll attitude, max. roll attitude is limited to } \\
35 \mathrm{deg}\end{array}$ & $\begin{array}{l}\text { Verify switching from rate to attitude } \\
\text { control, verify limits }\end{array}$ \\
\hline Load factor capture & $\begin{array}{l}\text { Pilot and automatic inputs, fly several } 360 \text { deg } \\
\text { turns ascending and descending (change flight } \\
\text { path angle with load factor command in be- } \\
\text { tween) }\end{array}$ & $\begin{array}{l}\text { Verify tracking and stability of load fac- } \\
\text { tor command, investigate flight path sta- } \\
\text { bility }\end{array}$ \\
\hline Automatic step inputs & $\begin{array}{l}\text { Step inputs are inserted into the system instead } \\
\text { of pilot stick force }\end{array}$ & $\begin{array}{l}\text { Generation of inputs with exact ampli- } \\
\text { tude and step width generates step re- } \\
\text { sponses in order to objectively quantify } \\
\text { controller performance }\end{array}$ \\
\hline $\begin{array}{l}\text { Configuration } \\
\text { changes }\end{array}$ & $\begin{array}{l}\text { Extension of flaps, gear during with no control } \\
\text { input, after transition to new configuration give } \\
\text { small inputs }\end{array}$ & $\begin{array}{l}\text { Verify controller robustness against } \\
\text { model uncertainties, change in aircraft } \\
\text { dynamics }\end{array}$ \\
\hline
\end{tabular}

the tested LPV method should provide robustness against model uncertainties, configuration changes were performed during flight to investigate stability during transition as well as responses after the configuration change.

\section{Longitudinal Flight Test Results}

Due to the autopilot pitch servo actuator having limited authority, the load factor reference controller is validated during a bank maneuver. The load factor command response is displayed in Fig. 8 . This setup allows load factor commands of multiple seconds to be fed without changing the aircraft's operating point in terms of airspeed and altitude. In Fig. 8 the pilot first banks to the left performing two steps and after approximately 10 seconds of steady turning, steps in bank attitude to the right are performed. The left upper plot of Fig. 8 shows the associated load factor demand and resulting load factor for the maneuver. It can be seen, that commanded load factor during the bank maneuver is tracked nicely by the pitch controller, keeping the flight path angle (left lower plot) at around zero degrees.

\section{Lateral Flight Test Results}

For analyzing the lateral tracking performance, small step inputs are used as verification. Fig. 9 shows an automatic step input given to the system and the respective response as well as control surface deflections. The left hand side of Fig. 9 shows roll and yaw rate as well as the reference signal for roll rate. The roll rate (variPhi) follows the step in expected PT-1 like behavior with a time constant of approximately $1 \mathrm{~s}$ as designed for the closed loop (see Fig. 4). Yaw rate $(\dot{\Psi})$, the second variable in the left hand picture, reacts without oscillations. Thus, the DR mode is sufficiently stabilized by the lateral MIMO control law. The right hand side plot shows the control surface deflections and angle of sideslip. As the lateral control law is designed for the multi input multi output problem with aileron and rudder as outputs, both control surfaces show deflections at the same time. In a conventional control law, the roll input would develop angle of sideslip first which would then be corrected by the yaw controller. In this case, rudder deflection follows immediately after initiating the roll maneuver by aileron input. Following, the developed sideslip has a maximum of $0.3 \mathrm{deg}$ after $4 \mathrm{~s}$ of the roll maneuver.

\section{E. Simulated Single Engine Failure}

Figure 10 shows the response of a simulated single engine failure in which the pilot manually decreases the power setting of one engine and at the same time increases the power of the remaining one in order to keep up constant level 

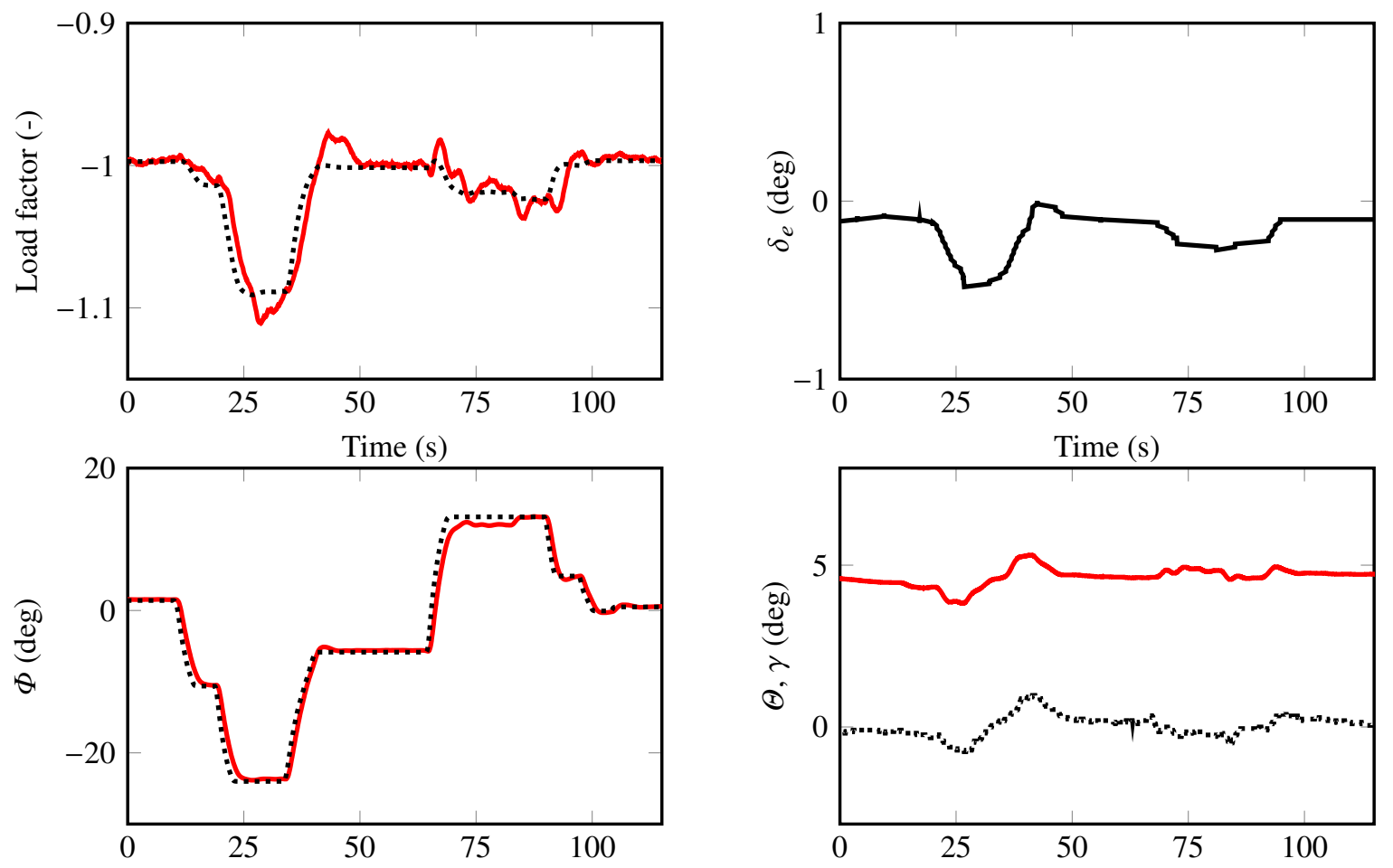

Fig. 8 Load factor response during a roll maneuver. The left lower plot shows integrated roll rate command and response, right lower plot shows pitch attitude $\square$ and flight path angle. The right upper plot shows elevator deflection relative to trim position.
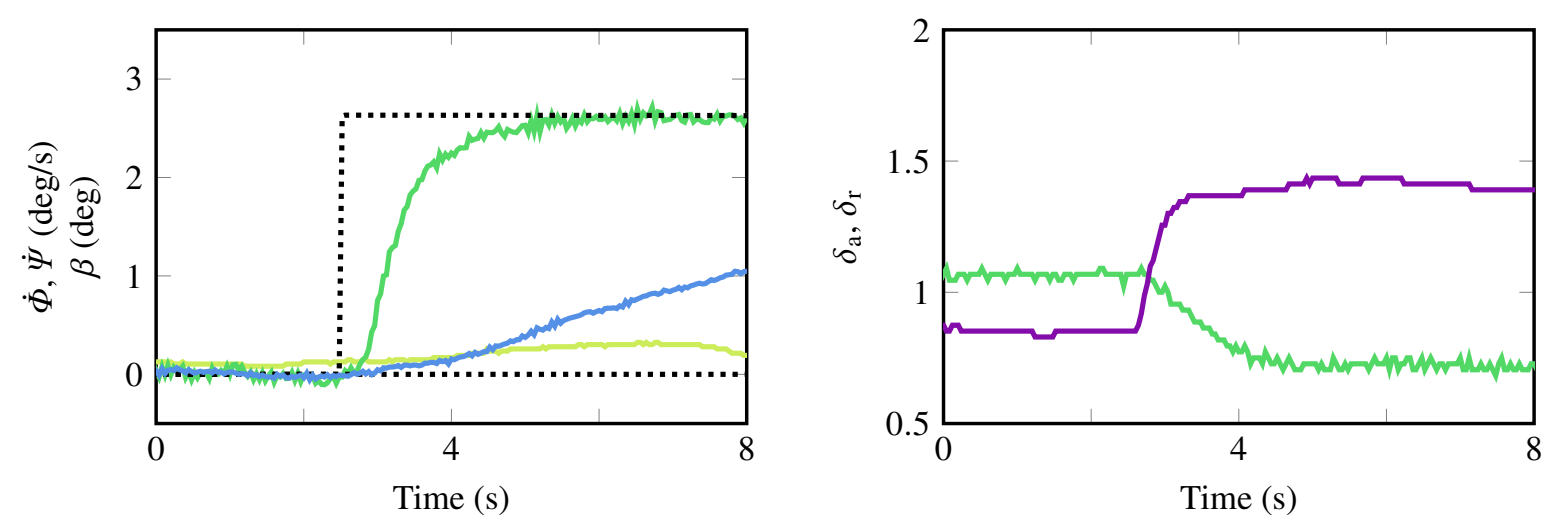

Fig. 9 Step response in roll rate. On the left hand side the responses in derivatives of bank angle $\dot{\Phi} \square$ and yaw angle $\dot{\Psi} \square$ are shown as well as angle of sideslip $\beta \square$. The right hand side plot shows deflections of ailerons $\square$ and rudder $\square$.

flying with constant indicated airspeed. The bottom left plot shows the actual engine power setting N1 whilst the top plots depict the response in angle of sideslip and roll attitude. The desired aircraft behavior is zero angle of sideslip as well as a steady roll attitude. Both goals are achieved by simultaneous deflection of rudder and aileron, which is the expected reaction of the lateral MIMO controller. With no angle of sideslip, the aircraft continues straight and level flying despite a differential thrust setting of $40 \%$ engine power on the left and $95 \%$ power on the right engine.

In addition to testing the different maneuvers listed in Table 3, the augmented inner loop control law was also enabled in the initial approach phase where aircraft configuration (flaps extended, gear down), deviated from the clean configuration used in synthesizing the LPV controller. As the pilot was capable of maneuvering without difficulties 

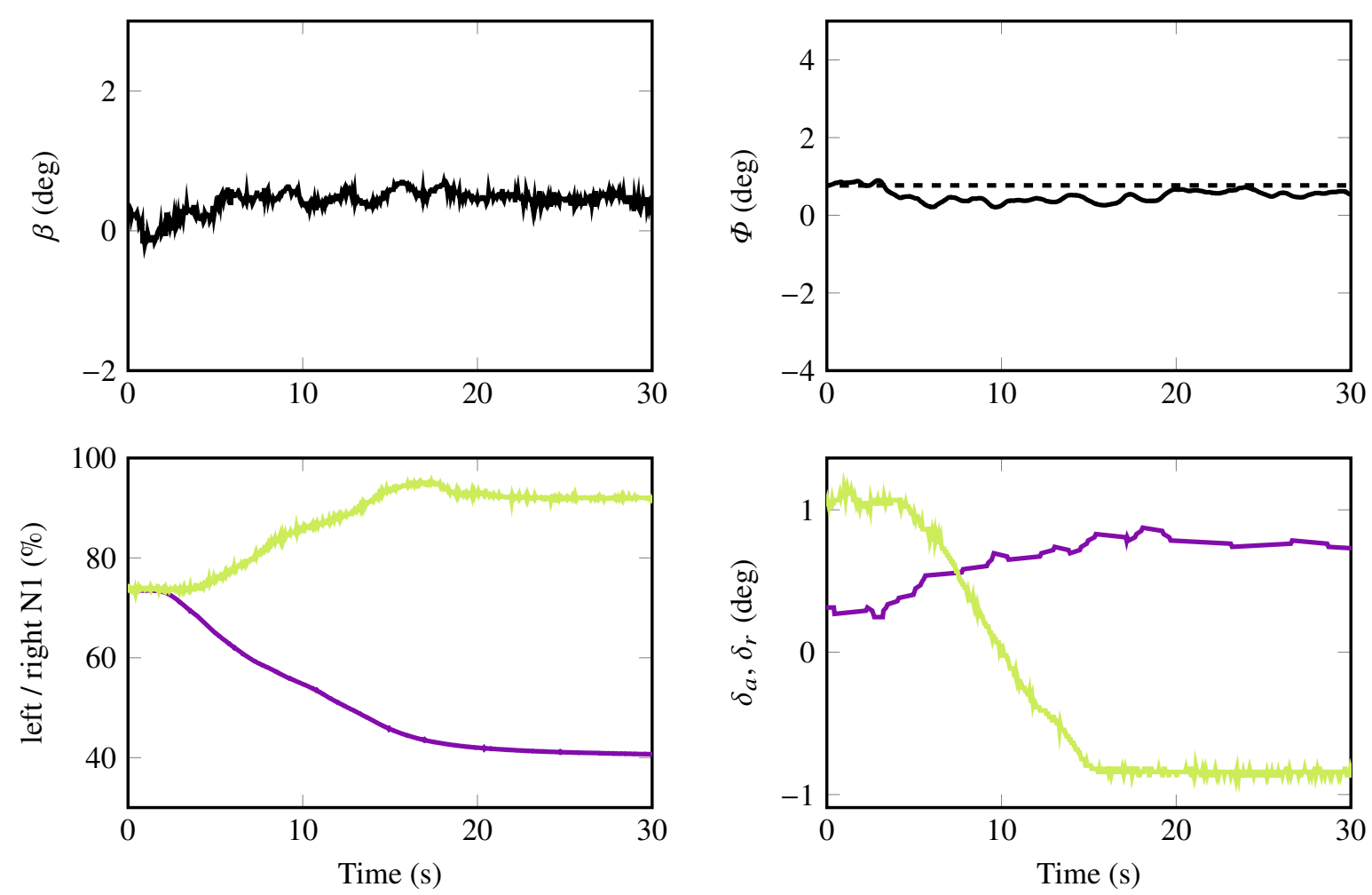

Fig. 10 Simulated single engine failure: responses in angle of sideslip $(\beta)$, bank angle $(\Phi)$, left $\square$ / right $\square$ engine setting and deflections of aileron $\square$ and rudder $\square$.

even in landing configuration, a qualitative evidence for robustness against changes in the aerodynamic characteristics of the aircraft is provided for the presented LPV control laws.

\section{Conclusions}

In this work, the results of an extensive flight test campaign to validate a linear parameter varying control system developed for a CS25 passenger aircraft have been presented. A selection of validation maneuvers in pitch and roll axis has been discussed. The tested flight control algorithms have been proven to provide very good handling qualities according to the test pilot's feedback. This has been backed with evaluation of test data. The gain scheduling feature of the controller, which comes naturally from the design process, has been verified in flight through maneuvering at different airspeeds. Finally, configuration changes were used for demonstration of satisfactory robustness of the control laws against unknown model changes.

\section{Acknowledgments}

The authors would like to thank the following persons from Delft University of Technology for their support: Olaf Stroosma for providing and helping with the DUECA implementation and GUI design; Menno Klaassen, for his assistance before and during the flight campaign; and Alexander in 't Veld and Tjipke van Netten for their role as test / safety pilots and also for giving valuable feedback on handling qualities of the controller. Further, the authors would like to thank their colleagues Tobias Bellmann, Andreas Seefried and Sebastian Kümper for their support conducting the Robotic Motion Simulator experiments in preparation of the flight campaign. 


\section{References}

[1] Papageorgiou, G., and Glover, K., "Design of a robust gain scheduled controller for the high incidence research model," Guidance, Navigation, and Control Conference and Exhibit, American Institute of Aeronautics and Astronautics, Portland, Oregon, 1999. doi: https://doi.org/10.2514/6.1999-4276.

[2] Papageorgiou, G., Glover, K., D’Mello, G., and Patel, Y., “Taking robust LPV control into flight on the VAAC Harrier," Proceedings of the 39th IEEE Conference on Decision and Control (Cat. No.00CH37187), Vol. 5, IEEE, Sydney, Australia, 2000, pp. 4558-4564. doi: https://doi.org/10.1109/CDC.2001.914633.

[3] Apkarian, P., Gahinet, P., and Becker, G., "Self-scheduled $\mathrm{H}_{\infty}$ control of linear parameter-varying systems: a design example," Automatica, Vol. 31, No. 9, 1995, pp. 1251-1261. doi: https://doi.org/10.1016/0005-1098(95)00038-x.

[4] Weiser, C., Ossmann, D., and Heller, M., "In-Flight Validation of a Robust Flight Controller Featuring Anti-Windup Compensation," 2018 Atmospheric Flight Mechanics Conference, American Institute of Aeronautics and Astronautics, Atlanta, Georgia, 2018. doi: https://doi.org/10.2514/6.2018-2982.

[5] Grondman, F., Looye, G., Kuchar, R. O., Chu, Q. P., and Van Kampen, E.-J., "Design and Flight Testing of Incremental Nonlinear Dynamic Inversion-based Control Laws for a Passenger Aircraft," 2018 AIAA Guidance, Navigation, and Control Conference, American Institute of Aeronautics and Astronautics, Kissimmee, Florida, 2018. doi: https://doi.org/10.2514/6.2018-0385.

[6] Pollack, T., Looye, G., and Van der Linden, F., "Design and flight testing of flight control laws integrating incremental nonlinear dynamic inversion and servo current control," 2019 AIAA Guidance, Navigation, and Control Conference, American Institute of Aeronautics and Astronautics, San Diego, California, 2019. doi: https://doi.org/10.2514/6.2019-0130.

[7] Keijzer, T., Looye, G., Chu, Q., and Van Kampen, E.-J., "Design and Flight Testing of Incremental Backstepping based Control Laws with Angular Accelerometer Feedback," 2019 AIAA Guidance, Navigation, and Control Conference, American Institute of Aeronautics and Astronautics, San Diego, California, 2019. doi: https://doi.org/10.2514/6.2019-0129.

[8] Weiser, C., Ossmann, D., Kuchar, R., and Looye, G., "Design and Verification of a Linear Parameter Varying Control Law for a Transport Aircraft," 5th CEAS Specialist Conference on Guidance, Navigation \& Control, Milan, Italy, 2019.

[9] Skogestad, S., and Postlethwaite, I., Multivariable Feedback Control: Analysis and Design, $2^{\text {nd }}$ ed., Wiley, Chichester, 2005.

[10] Wu, F., “Control of Linear Parameter Varying Systems,” PhD Thesis, University of California, Berkely, 1995.

[11] Pfifer, H., LPV/LFT Modeling and its Application in Aerospace, $1^{\text {st }}$ ed., Luftfahrt, Dr. Hut, München, 2013.

[12] US Department of Defense, MIL-F-1797C: Flying Qualities of Piloted Aircraft, 1997.

[13] Wu, F., Yang, X. H., Packard, A., and Becker, G., "Induced $\mathrm{L}_{2}$-norm control for LPV systems with bounded parameter variation rates," International Journal of Robust and Nonlinear Control, Vol. 6, No. 9-10, 1996, pp. 983-998. doi: https://doi.org/ 10.1002/(sici)1099-1239(199611)6:9/10<983::aid-rnc263>3.0.co;2-c.

[14] Pfifer, H., and Hecker, S., "LPV controller synthesis for a generic missile model," 2010 IEEE International Conference on Control Applications, IEEE, Yokohama, Japan, 2010, pp. 1838-1843. doi: https://doi.org/10.1109/CCA.2010.5611127.

[15] Hjartarson, A., Seiler, P., and Packard, A., "LPVTools: A Toolbox for Modeling, Analysis, and Synthesis of Parameter Varying Control Systems," IFAC-PapersOnLine, Vol. 48, No. 26, 2015, pp. 139-145. doi: https://doi.org/10.1016/j.ifacol.2015.11.127.

[16] van der Linden, C. A. A. M., Dasmat-Delft University Aircraft Simulation Model and Analysis Tool: A Matlab, Simulink Environment for Flight Dynamics and Control Analysis (Series 03 - Control and Stimulation, No 03), Delft Univ Pr, 1998.

[17] van den Hoek, M. A., de Visser, C. C., and Pool, D. M., "Identification of a Cessna Citation II Model Based on Flight Test Data," Advances in Aerospace Guidance, Navigation and Control, Springer International Publishing, Cham, 2018, pp. $259-277$. doi: https://doi.org/10.1007/978-3-319-65283-2_14.

[18] Braven, S. d., and Verspay, J., "Identification of the Servo Actuated Flight Control System of the Cessna Citation II," Tech. Rep. VM-94006, National Aerospace Laboratory NLR, 1994.

[19] Brockhaus, R., Alles, W., and Luckner, R., Flugregelung, $3^{\text {rd }}$ ed., Springer-Verlag Berlin Heidelberg, Berlin Heidelberg, 2011.

[20] Schug, A.-K., Seiler, P., and Pfifer, H., "Robustness Margins for Linear Parameter Varying Systems," AerospaceLab, Vol. 13, 2017. doi: https://doi.org/10.12762/2017.AL13. 
[21] Bates, D., and Postlethwaite, I., Robust Multivariable Control of Aerospace Systems, Control And Simulation, DUP Science, 2002.

[22] Zaal, P., Pool, D., in 't Veld, A., Postema, F., Mulder, M., van Paassen, M., and Mulder, J., "Design and Certification of a Fly-by-Wire System with Minimal Impact on the Original Flight Controls," AIAA Guidance, Navigation, and Control Conference, American Institute of Aeronautics and Astronautics, Chicago, Illinois, 2009. doi: https://doi.org/10.2514/6.2009-5985.

[23] European Aviation Safety Agency, Certification Specifications and Acceptable Means of Compliance for Large Aeroplanes CS-25, 2018.

[24] Muis, A., Oliveira, J., and Mulder, J. A., "Development of a flexible flight test instrumentation system," 17th SFTE (EC) Symposium, Amsterdam, Netherlands, 2006.

[25] Lu, P., Van Eykeren, L., Van Kampen, E.-J., De Visser, C., and Chu, Q., "Aircraft Inertial Measurement Unit Fault Identification with Application to Real Flight Data," Journal of Guidance, Control, and Dynamics, Vol. 38, 2015, pp. 2467-2475. doi: https://doi.org/10.2514/1.G001247. 